\title{
La Educación: Lo Público y Lo Político ${ }^{1}$
}

\author{
Education: Public And Political
}

\author{
Rodrigo Baño Ahumada \\ Especialista en Sociología Política \\ Departamento de Sociología \\ Facultad de Ciencias Sociales \\ Universidad de Chile \\ Av. Capitán Ignacio Carrera Pinto 1045, Nuñoa \\ rbano@uchile.cl
}

\begin{abstract}
Resumen: Se pretende tratar de aclarar algunos aspectos básicos respecto al sentido e importancia de la educación, el significado de lo público y el sentido político de toda propuesta que se hace al respecto. El tema de la educación es primeramente político y empieza por la definición del carácter del Estado. De la definición del Estado como subsidiario se deriva la comprensión de la educación como un bien de consumo que se transa en el mercado en la cantidad y calidad que corresponda al respectivo poder de compra. De la definición de un Estado responsable deriva la comprensión de la educación como un derecho. Es la voluntad política la que establece una u otra definición.
\end{abstract}

Palabras clave: Educación - Estado - Público

Abstract: The aim of this paper is to clarify some basic aspects concerning the purpose and importance of education, the meaning of what is public, and the political significance of all proposals presented regarding education. The issue of education is primarily political and begins with how the character of the State is defined. On one hand, if the State has a subsidiary definition, education is considered to be a commodity that is negotiated in the market in the appropriate quantity and quality which correspond to the respective purchasing power. On the other hand, if the State is defined as responsible, education is considered to be a right. Political will is what establishes one definition or the other.

Keywords: Education - State - Public

Aunque todos lo sabemos, solemos olvidar que la realidad concreta es un continuo heterogéneo del cual abstraemos analíticamente determinados aspectos que elaboramos conceptualy teóricamente. Pero toda abstracción corre siempre el peligro de la reificación, es decir, de pensar y tratar las abstracciones como si se tratara de objetos reales. Es lo que ocurre frecuentemente con el concepto Estado, que deja de ser una abstracción conceptual para ser identificado con el personal burocrático o las oficinas públicas. A su vez, la separación analítica de un objeto suele olvidar su carácter de tal y conducir a la idea de una separación real, aislándola completamente de su relación con la 
totalidad de la que forma parte. Esto suele conducir a la explicación endógena de las abstracciones que se han construido, olvidando sus relaciones con el entorno. Talvez por eso me atrevería a plantear al inicio una consideración, que se pudiera llamar de contexto, antes de abordar el tema de la educación en general, de su carácter público y de las confusiones que al respecto se plantean; con especial referencia a las universidades.

La educación siempre ha sido tema de preocupación en todas las sociedades que se conocen, pues es la forma en que éstas se mantienen y reproducen. Se transmiten los conocimientos y las pautas de orientación valorativas, aunque esto se realice inicialmente de manera intuitiva, Las sociedades no viven sin historia, así como los sujetos individuales no viven sin memoria. Considerando, además, que el pasado no es antecedente del presente, sino que es su componente: el adulto no deja de ser el niño que fue, así como las sociedades no dejan de ser el pasado que tuvieron. Pero la educación no apunta sólo a la reproducción de las sociedades y de los individuos, sino que también establece los fundamentos de la proyección de sociedades e individuos. Historia y utopía.

En la medida que las sociedades pierden su ingenuidad y toman conciencia y racionalizan sus acciones, la educación pondrá más el acento en el proyecto que en la historia. El pensamiento clásico griego y especialmente Platón desarrollará la convicción de que el ser humano tiene plasticidad para ser moldeado, que puede formarse al ciudadano en una orientación determinada. Gran parte de La República de Platón está destinada a explicitar el hecho de que la educación es el instrumento para moldear el buen ciudadano.

Las instituciones educacionales de la época moderna tienen el mismo supuesto, esto es, que es posible formar al ser humano de determinada manera. Dichas instituciones se desarrollarán entonces con esa pretensión de formar, pero es necesario tener en cuenta que también históricamente cambiará la determinada manera en que se pretende formar. Consecuentemente, el ciudadano que se pretende formar en la Grecia Clásica será distinto al que se intenta formar en la Edad Media y al que se intenta formar en los siglos XIX, XX y XXI.

Dentro de esas instituciones formadoras se irá escalando en contenido y profundidad hasta llegar a las universidades, las que ya cuentan con una larga historia. Todos sabemos que las universidades existen desde la baja edad media y que algunas de las actualmente existentes en Europa fueron creadas en el siglo XII y especialmente en el siglo XIII, mientras que en América española empezaban ya a fundarse en el siglo XVI. No obstante, también 
todos comprenden que aquellas universidades son muy distintas de las que actualmente existen y eso da buena cuenta de que, aunque persista la idea de que es posible formar al ser humano a través de la educación, esto es, orientar valorativamente a los sujetos y entregarles conocimientos que los habiliten para realizar su función social, las maneras y orientaciones de esa formación han ido cambiando.

Los cambios que ocurren en las universidades no se producen en el vacío, ni son un desarrollo endógeno de posibilidades inscritas en su esencia, sino que corresponden a las transformaciones sociales que se van produciendo Los distintos modelos de universidades que se irán desarrollando a lo largo del tiempo irán planteando de manera también distinta esos dos objetivos educacionales en el más alto nivel de estudios, especialmente en el modelo de universidad que inspiró a las nuestras, en que se asigna a las universidades no sólo la transmisión de conocimientos, sino también la creación de ellos mediante la investigación. Como nos suelen recordar quiénes se preocupan del tema, es el desarrollo de las universidades nacionales el que irá generando distintas tradiciones universitarias, como ocurre en Francia, Alemania, Estados Unidos y como ocurrirá en América española a partir del modelo de las universidades castellanas. Más allá de las diferencias que presentan esas tradiciones, la universidad seguirá siendo concebida como la institución orientada especialmente a la creación y comunicación de conocimientos de más alto nivel.

Si saltamos con cierta brusquedad a la situación del siglo XX recién pasado, nos vamos a encontrar con una serie de propuestas y movimientos destinados a cambiar el carácter de las universidades prevaleciente en América Latina y particularmente en Chile. En efecto, en la medida que empieza a plantearse social y políticamente el tema del cambio social, la universidad será revalorada en términos de aquel viejo problema de cómo educar en el más alto nivel para entregar conocimientos y orientación de valores que impulsen el tipo de cambio social deseado. La preocupación práctica, de política educacional, pondrá entonces el énfasis en estudiar la forma en que la universidad puede impulsar el cambio social; qué reformas y adecuaciones requiere para servir a los propósitos que se desea impulsar y surgirán distintos proyectos y proposiciones de diverso desenlace en aquella época.

Talvez sería conveniente recordar que las reformas universitarias producidas en nuestra región, no sólo responden a las orientaciones ideales que plantearon sus impulsores, sino que se relacionan con fuertes cambios producidos a nivel de sociedad. En efecto, las reformas propuestas a partir de 
aquella famosa ocurrida en Córdoba en 1918, dan cuenta de una crisis de la dominación oligárquica y del ascenso de otros sectores sociales que ponen en cuestión los estilos y orientaciones de la vieja universidad; no son simplemente la puesta en práctica de un desarrollo autónomo de las ideas. De la misma manera, las reformas universitarias de la década de los sesenta no sólo son expresión de la onda expansiva que adquieren movimientos estudiantiles generados en los países centrales, sino que se corresponden aquí a una nueva crisis de un modelo socioeconómico que frustra las aspiraciones de ascenso social de las capas medias y a la crisis de un modelo político sobrepasado por la participación popular. En ambos casos, aunque las reformas producidas apuntaron fundamentalmente a aspectos de organización y procedimientos de las universidades, pareciera que la principal modificación real apuntaba al sentido de reorientación ética y operativa de la educación universitaria.

Cuando en el momento actual nos volvemos a plantear el problema universitario, talvez sería conveniente revisar no sólo lo que nos pueda parecer el mejor modelo de universidad o de sistema universitario, sino que atender a cuáles son las condiciones sociales que generan la problematización de las universidades. Porque si ahora nos preocupa la situación de la universidad, en cuanto universidad pública, debemos considerar que este no fue un problema en América Latina durante mucho tiempo, ya que lo natural y obvio es que las universidades fueran públicas, estatales, aunque se mantuvo la presencia de universidades católicas. La emergencia y crecimiento de las universidades privadas no es un dato casual, no corresponde a un desarrollo autónomo de las instituciones, sino que está relacionado con un cambio estructural e ideológico en nuestras sociedades.

Como es bien sabido, pero es bueno repetirlo, en Chile la gran transformación en el sistema educacional en general y en las universidades en particular se producirá bajo el gobierno del general Pinochet. Pero la gran transformación no es sólo del sistema educacional, sino que todo el sistema socioeconómico y político sufrirá un espectacular cambio. De este cambio quisiera resaltar fundamentalmente el cambio en la consideración del papel del Estado y su relación con el mundo privado. El Estado es definido en términos estrictos de Estado subsidiario y, por tanto, transfiere a los particulares todo lo que estos quieren y pueden realizar. De un modelo en que el Estado se concebía como órgano mediador de los intereses considerados en el compromiso social que lo sustentaba, asumiendo funciones de protección social sobre la base de la consideración de derechos, se pasará a un sistema fuertemente privatizado. Esto se manifestará claramente en la educación, que perderá su carácter 
esencialmente público, financiado y provisto por el Estado, para pasar a privilegiarse la empresa privada de educación.

El Estado se desprende de la propiedad y administración de los centros educacionales y sólo admite una función subsidiaria. La educación básica y media está entregada a la gestión empresarial de municipios y particulares que quieran hacerse cargo de ella a cambio de la venta del servicio a quiénes lo requieran. Para el caso de quiénes no están en condiciones de pagar esa educación, el Estado subsidia a las corporaciones que se hacen cargo de ella, sean estos particulares o municipales.

Respecto de las universidades, aunque el Estado mantiene un pequeño aporte directo a las públicas y privadas tradicionales (en la estatal Universidad de Chile es de aproximadamente el 10\% de su presupuesto y en otras estatales es aún inferior), todas son pagadas por quiénes estudian en ellas. Como se suele señalar, la educación chilena es la más cara del mundo, pues la educación superior se lleva el 22\% de los ingresos de los hogares. El Estado, con su rígida definición de subsidiario, otorga becas a los más pobres y otorga crédito para que paguen a los que son un poco menos pobres. Aunque subsiste la denominación de universidades públicas o estatales, como la Universidad de Chile y la Universidad de Santiago (anterior Técnica del Estado), además de las sedes regionales de éstas y el antiguo Pedagógico de la Universidad de Chile, que fueron transformadas en universidades distintas, todas han sido en los hechos privatizadas, ya que la necesidad de autofinanciarse obliga a que cobren cuantiosos aranceles a sus estudiantes y procuren vender servicios de todo tipo.

Puede resultar extraño que, después de más de veinticinco años del retorno a la democracia en Chile y de las grandes esperanzas que ello suscitó, todavía nada de esto ha cambiado y sólo recientemente se empieza a discutir un posible cambio, cuyos resultados son bastante impredecibles, ante la arremetida política e ideológica de los intereses creados con el actual modelo. Las grandes movilizaciones sociales que han ocurrido en Chile en el último tiempo son las que han posibilitado esta apertura a una posible reforma y ellas se comprenden perfectamente con estos antecedentes. Se trata de movilizaciones políticas, no en el sentido de que sean impulsadas por algún partido o agrupación política, sino porque se corresponden con el elemento que es esencial en la política, que es el conflicto por la igualdad. Al efecto, lo que el movimiento por la educación reclama es la consideración real de la educación como un derecho, lo que significa que el Estado debe garantizarlo a todos en condiciones de igualdad. 
Esta afirmación, de que la educación es un derecho, estuvo en la base de la organización de la educación en Chile, especialmente resaltada a partir de la década de los treinta cuando se explicitó tajantemente que el Estado tenía el carácter de Estado docente. Existe todavía la consagración constitucional del derecho a la educación, pero este derecho aparece sin garantía. La educación básica y media dependiente de corporaciones municipales es la única gratuita para sus alumnos, pero ha sido abandonada a las peores condiciones y es reconocidamente de mala calidad, constituyéndose en un obstáculo para acceder a niveles superiores de educación. Quiénes pueden ingresan a sus hijos en establecimientos particulares subvencionados, donde pueden hacer un copago para que mejore un poco el nivel educacional. Quiénes tienen dinero suficiente matriculan a sus hijos en colegios particulares pagados de alto costo y mejor calidad. El sistema funciona coherentemente como reproducción de la situación social de los padres.

En tales condiciones no sorprende que el entonces Presidente Piñera, ante las demandas estudiantiles por una educación pública gratuita de buena calidad, haya proclamado enfáticamente que la educación es un bien de consumo. De manera que aparece negado lo que anteriormente se consideró obvio, que la educación es un derecho, para explicitar la consideración contraria con el mismo énfasis de obviedad.

La afirmación de que la educación es un bien de consumo, implica que es un bien que se produce para que los consumidores puedan tener acceso a ella a través del mercado, regulado según la oferta y demanda de educación. El ajuste entre oferta y demanda se realiza a través del precio, que determina que se pueda acceder a la educación según el poder de compra de cada uno. Naturalmente el precio puede variar dependiendo de la cantidad y calidad de este bien, de manera que el consumidor, según sus necesidades y poder de compra, adquirirá el que esté a su alcance.

Lo que quizás podría resultar sorprendente, para los que creen en la obviedad de que la educación es un bien público, es comprobar que el modelo educacional chileno se adapta fielmente a la consideración de la educación como un bien de consumo. En efecto, hay empresas de educación que ofrecen su producto a un mercado de consumidores dispuestos a pagar, según su poder de compra, por el suministro del servicio en la cantidad y calidad que ese poder de compra y sus necesidades determinan. Naturalmente esto implica considerar que la necesidad de educación es considerada en términos del interés personal de realizar una inversión que permita posteriormente obtener un beneficio económico de ella. Sin embargo, hay que aclarar que, aún en este 
modelo, el Estado asume que la educación es importante para el conjunto de la sociedad y declara a ésta obligatoria hasta cierto grado, contribuyendo a proveer este servicio mediante subsidios a los sostenedores municipales o particulares de instituciones de educación que entregan la calidad y cantidad que tales subsidios permiten. En la educación superior esto se expresa mediante la entrega de becas y préstamos a los que no pueden comprar el servicio. Los que poseen mayor poder de compra pueden concurrir a la adquisición de los servicios de mayor calidad.

De manera que la expectativa de derecho empieza a derrumbarse, pues se hace evidente que la afirmación de que la educación es un bien público tiene más un carácter de fantasía que de realidad. El Estado se limita a cubrir malamente la educación básica y media que declara obligatoria, mientras la de buena calidad se transforma en un privilegio para el que pueda pagar. Después de décadas de aplicación del modelo privatizador los ciudadanos se resignan a esto y la fundamentación ideológica empieza a transformarse en sentido común. Se devalúa la consideración de derechos y se plantea como un absurdo que el Estado entregue educación gratuita a los que pueden pagarla, aceptándose sólo el aporte piadoso de que se la pague a los vulnerables que no tienen el dinero necesario.

Lo anterior no resulta tan extraño si se piensa en términos más genéricos, puesto que es una característica de los últimos tiempos la preeminencia que adquiere lo privado sobre lo público y la tendencia a desplazar las coordinaciones sociales desde el Estado hacia el mercado. No es extraño, entonces, que cualquier argumentación a favor de lo público sea regularmente tildada de actitudes nostálgicas de quiénes no perciben ni aceptan las profundas transformaciones que ha experimentado la humanidad. Más aún, si se sigue manteniendo una valoración de lo público, ello se hace asumiendo que lo público no está en el Estado, llegándose al absurdo de proclamar que los establecimientos de educación privados son públicos y, a la vez, que los del Estado deben ser tratados como los privados.

Parece evidente que los cambios de modelo económico, tanto a nivel nacional como a nivel global, no sólo han sido impulsados por una nueva hegemonía social, sino que han generado también transformaciones sociales profundas y asentado nuevas orientaciones ideológicas. La diferenciación público privado ha adquirido otras connotaciones y el papel del Estado también ha adquirido otro carácter. 
Aunque perezca una ingenuidad, puede ser conveniente empezar preguntándose cuándo empezó todo esto y qué le pasó en el camino. El recurso a los orígenes como factor explicativo mantiene su potencia a pesar de la pedantería posmoderna de pretender una comprensión sin memoria y sin historia. Así, la diferenciación entre lo público y lo privado marca en su origen el significado de estos conceptos.

No es extraño que los autores modernos recurran a los clásicos para enfrentar las confusiones que se han derivado de las primeras conceptualizaciones. Es lo que ocurre con el análisis que se hace de la muy mentada frase aristotélica de que el hombre es un animal político. Como es sabido, no se pretende en esta frase apuntar al carácter social del ser humano, pues animales sociales hay muchos, sino que, como señala el mismo Aristóteles, el hombre es el único animal que tiene el uso de la palabra, lo que le permite distinguir lo justo de lo injusto y, por tanto, vivir bajo normas elaboradas por él mismo; eso es lo que lo hace ser el único animal político. Es el único animal que hace eso. A partir de aquí es que se hace la distinción entre el ámbito de lo público, donde se realiza la política que elabora las normas, y el ámbito de lo doméstico, donde se realiza el trabajo para la satisfacción de las necesidades. Lo público se realiza en el plano de la igualdad de los ciudadanos; lo doméstico se realizaba en el plano de la desigualdad propia de la organización del hogar, especialmente marcada en aquellos tiempos. Es un punto de partida que no se debe olvidar pues se continúa en el significado de la República: la res pública romana es lo común a todos considerados en su igualdad de ciudadanos.

Desde aquellos tiempos clásicos, una de las transformaciones de mayor importancia es la que se ha venido desarrollando por el paso de la economía doméstica a la economía política. De hecho la expresión economía política es una contradicción en los términos, puesto que oikos, desde donde deriva economía, es la comunidad familiar en que se producía lo necesario para la supervivencia, mientras que la polis es la comunidad de ciudadanos libres, ajenos al mundo de las necesidades, que definen los destinos de la comunidad.

Históricamente se desarrolla un largo proceso, que llega hasta nuestros días, en que las actividades de producción que se realizaban en el ámbito doméstico, en la casa, pasan a ser realizadas externamente. Algunos recordarán, o habrán escuchado, que no hace tanto tiempo que todavía en el hogar se realizaba una cantidad importante de actividades de producción de bienes y servicios: huertos familiares, crianza de animales para el consumo, elaboración de alimentos, conservas, confección y reparación de ropa, cuidado de los niños, producción de juegos y entretenciones, reparaciones del hogar y muchas otras. En la actualidad 
casi toda esa producción doméstica se ha externalizado, lo que se relaciona muy directamente con el desarrollo capitalista, que ha incorporado a la fuerza de trabajo empresarial a quiénes realizaban estas actividades domésticas, a la vez que produce para el mercado los bienes y servicios que anteriormente se producían en el hogar. Es el desarrollo de la sociedad moderna, que ha generado una muy compleja red de relaciones en que los diferentes agentes buscan la satisfacción de sus particulares intereses. En la medida que esta red se hace cada vez más compleja, los individuos se hacen cada vez más dependientes de ella, al punto de que carecen de toda posibilidad de sobrevivencia sin ella. El orgulloso individuo libre contemporáneo es incapaz de generar sus mínimas condiciones de existencia. Se podría decir que nunca la producción ha sido tan social como ahora y, por lo tanto, la economía tan política.

Esta compleja red de relaciones interpersonales, que pudiera considerarse como una abrumadora expansión de la sociedad civil, implica, casi paradojalmente, también una abrumadora expansión del Derecho y del aparato burocrático del Estado, puesto que todas estas nuevas actividades requieren ser controladas a fin de que los sujetos puedan realizar sus interrelaciones con un mínimo de seguridad que las haga posibles. Nadie espera que el estricto funcionamiento de las reglas del mercado, necesariamente imperfecto por lo demás, permita garantizar la seguridad de los contratos, la salud pública, la adecuada información etc. La confianza que generaba la economía doméstica, al unificar al productor con el consumidor, se desvanece en la medida en que la producción se desarrolla en función de la ganancia. El Estado es el único que puede restablecer esa confianza al garantizar coactivamente que se cumplan determinadas condiciones en la producción y distribución de esa producción social.

Por otra parte, el Estado, como el lugar de lo público en el mundo moderno, establece la definición de lo común, del interés general de una sociedad y sobre esta base reconoce derechos y obligaciones de ese carácter. El Estado, principio de totalidad social y voluntad de esa totalidad, es el lugar de la igualdad, independiente de las desigualdades que generan las actividades destinadas a la satisfacción de necesidades. En tal sentido, el Estado moderno establece una burocracia profesional y un derecho que se define a partir del reconocimiento de la igualdad de los ciudadanos. Esta es la base del establecimiento de derechos, los que, en principio reconocen esta igualdad. Ante esta moderna polis los ciudadanos son libres e iguales, aunque no lo sean en el ámbito de las necesidades.

Lo anterior puede servir de introducción para ir despejando ciertas confusiones que conducen a elaboraciones ideológicas que en los últimos años han ido adquiriendo tal predominio que, como señalábamos, casi se plantean 
como sentido común. En efecto, el Estado tiende a ser presentado cada vez más como el lugar de la dominación, mientras que la sociedad civil suele ser percibida como el lugar de la libertad. De esto deriva la pretensión tantas veces planteada de que es necesario disminuir el Estado y aumentar la sociedad civil. Más aún, la actual demanda por la expansión de las ciudadanías, se presenta como si fuera una demanda de la sociedad en contra del Estado, puesto que se reclama el reconocimiento de "derechos" de los ciudadanos frente al poder público. Sin embargo, la demanda por ciudadanía y por derechos es una demanda política con pretensiones de constituirse en un valor y norma del mismo carácter, asumidos como expresión de la voluntad del Estado. No hay derechos sin garantía estatal.

Las confusiones, como suele ocurrir, arrancan de una ambigüedad semántica en el término Estado. Tal término se utiliza muchas veces para hacer referencia al aparato burocrático del Estado o, más restringidamente aún, al gobierno. Pocas veces se tiene conciencia de que el Estado es el principio de totalidad de una sociedad determinada, esto es, la voluntad de ese todo. El Estado es la voluntad general que delimita lo que es común y particular, las orientaciones de acción prescritas o proscritas. No obstante, hay que aclarar que definir el Estado como principio de totalidad social nada nos dice respecto a cómo se forma esta voluntad social, ni a qué clase, sector o grupo social expresa o beneficia. Muchas veces se ha señalado que en la constitución de los primeros estados la voluntad de totalidad es la voluntad del príncipe o el monarca; también se ha señalado, por el pensamiento socialista y marxista, que en el Estado capitalista la voluntad del todo es la que corresponde a los intereses de la burguesía; la tradición clásica, readecuada miles de veces, sostendrá que la voluntad de la totalidad se sustenta en la razón, que es la esencia de lo humano; la actual teoría de la democracia representativa propondrá procedimientos para asegurar que se extienda la participación de los individuos. El tema es muy complejo y requeriría un tratamiento especial, pero lo que aquí se quiere resaltar es que la afirmación de que el Estado es principio de totalidad social, voluntad política, no implica nada respecto a su evaluación positiva o negativa ni la relaciona necesariamente con algún fundamento o predominio social. De hecho, el que se esté hablando actualmente de una crisis de representación algo tiene que ver con la consideración de que la formación de la voluntad del Estado no pareciera corresponderse con lo que es su fundamento de legitimidad en la teoría democrática todavía vigente, esto es, la voluntad general.

Al analizar el desarrollo de la modernidad y la interpenetración de lo económico y lo político, muchos sustentan la afirmación de que se habría producido una fusión entre lo público y lo privado, en el sentido de que el 
Estado se habría socializado y que a su vez la sociedad se habría estatizado, de manera que lo público no reconocería actualmente esa diferenciación entre Estado y sociedad, sino que a lo público sólo se opondría actualmente lo íntimo. A la vez, se agrega a esta consideración todo tipo de análisis relacionados con el denominado proceso de globalización, que destruiría la antigua concepción del Estado para poner énfasis en la cada vez mayor interdependencia de los antiguos estados nacionales, lo que plantearía que las antiguas consideraciones del Estado estarían obsoletas. Lo cierto es que no pueden eludirse las transformaciones experimentadas, pero, más allá de esos cambios y de las tendencias que puedan observarse, el concepto de Estado sigue siendo plenamente vigente para el análisis político, pues seguimos viviendo en un mundo donde los estados son la principal institución y lo público sólo tiene sentido en referencia a ellos. Más aún, toda la gran transformación experimentada en el modelo económico desde fines del siglo XX no habría sido posible sin la notable intervención del Estado en esa dirección. No cabe ninguna duda de que el modelo neoliberal aplicado rigurosamente en Chile sólo fue posible mediante una fuerte intervención estatal que generó las condiciones para que ello fuera posible y que ha tenido una activa participación en su mantenimiento y profundización.

El Estado en la actualidad sigue siendo concebido como la expresión del interés general, pero junto a ese interés general o común a la totalidad social, está la multiplicidad de intereses particulares a los que se hacía referencia al mencionar el desplazamiento de la economía doméstica hacia la economía política. Precisamente en la llamada economía política lo que se tiene es la visión general de la red de relaciones entre intereses particulares y se procura desarrollar la perspectiva de lo público respecto a tales actividades económicas. Sin embargo, aunque parezca innecesario, es conveniente aclarar que lo público no es una sumatoria de lo particular, sino que es un ámbito o esfera completamente distinta que responde a criterios también distintos que impiden, por contradictorio, que se pueda producir una mercantilización de lo público, puesto que la mercantilización implica su privatización.

Es sabido que se han desarrollado definiciones muy variadas respecto del término público y actualmente es casi un lugar común la afirmación de que lo público no se identifica con lo estatal. Que algo sea público puede significar que involucra a un número significativo de personas o que meramente trasciende la esfera de lo íntimo. En tal sentido se puede hablar de un espectáculo público, de un mercado público o de una calamidad pública. No obstante, el Estado constituye lo público en su sentido más profundo, en cuanto da cuenta de lo común a una sociedad determinada y particularmente es la voluntad de esa totalidad. Ninguna otra entidad puede pretender ser esa voluntad del todo social, 
a menos que tenga la capacidad de eliminar el Estado y reemplazarlo. Cosa muy distinta es considerar que algo involucra a una gran cantidad de personas y emplear el término público para referirse a ello, de hecho muchas actividades privadas interesan o involucran a un gran número de personas e incluso a todas las personas de una sociedad determinada nacional o internacionalmente. Pero la polisemia del término no debe conducirnos a confusiones.

Dado que es el mismo Estado el que establece los límites entre lo público y lo privado, lo que es determinado por la voluntad del todo social y lo que es determinado por la voluntad de uno o varios sujetos, a la vez que define sus objetivos y procedimientos, lo que ha estado ocurriendo en los últimos decenios es que el Estado ha ido reduciendo el ámbito de lo público y ampliando la esfera privada. La ideología del Estado subsidiario apunta precisamente a establecer que el Estado sólo debe asumir como público lo que no puede o no quiere ser asumido directamente por los particulares, abriendo espacios para la acción de los privados e incluso transfiriéndoles recursos para garantizar el éxito de sus gestiones. Más aún, podría decirse que una de las claves del desarrollo del actual modelo económico radica en la transferencia de recursos que hace el Estado a la empresa privada, recursos que naturalmente se obtienen del pago de impuestos de los ciudadanos. En Chile, para el despegue del modelo económico, no sólo transfirió a la empresa privada la recaudación de la cotización obligada de los trabajadores para su previsión, sino que realiza enormes transferencias de recursos a las empresas privadas de salud, educación, trasportes, vialidad, construcción, etc., garantizando así las necesidades de acumulación y ganancia de éstas. Naturalmente los recursos que transfiere provienen del aporte impositivo de los ciudadanos. Sería interesante hacer un cálculo de las transferencias que directa e indirectamente realiza el Estado a las empresas a partir de los fondos recaudados por impuestos.

La reducción de lo público es actualmente muy notoria en el tema que nos ocupa aquí, esto es, la educación. En Chile el Estado ya no se hace cargo de la educación, sino que hasta la enseñanza media subvenciona a quiénes se hacen cargo de ella, sean estos corporaciones municipales o particulares, mientras que desaparecen de hecho las instituciones públicas de educación superior, sea ésta universitaria o técnica profesional, y sólo se provee con fondos públicos becas y créditos para los más necesitados que acceden a ella. Muchas subvenciones, becas y créditos estatales constituyen claramente una transferencia de recursos públicos a empresas privadas de educación.

No obstante esta privatización, el Estado no puede desentenderse totalmente de la educación, pues ésta se relaciona muy directamente con el 
interés general de la sociedad, expresada en la realización de un determinado proyecto, dado que la reproducción social está comprometida con la educación de las nuevas generaciones. No es casual, entonces, que la preocupación por la educación haya tenido siempre una alta consideración en la acción política. En la medida que la labor formativa trascendió el ámbito doméstico, fue el Estado quién asumió el papel principal en la educación de sus ciudadanos, aunque inicialmente su fusión con la Iglesia delegue en ésta esa tarea, para, posteriormente, asumirla directamente en plenitud. Prácticamente en todos los países el Estado ha asumido la educación formal como una de sus funciones esenciales, reconociendo que hay un interés general comprometido en ello, tanto por la formación ciudadana requerida como por las necesidades de capacitación laboral para el funcionamiento de la economía. La educación constituirá la base de la comunidad nacional y de la legitimidad en el Estado moderno, lo cual justifica su carácter obligatorio. Todo esto concluye en el reconocimiento formal de la educación como uno de los derechos fundamentales de los ciudadanos.

Que exista un interés general comprometido en la educación no significa que no existan intereses particulares en educadores y educandos. En la medida que la educación pasa a constituirse en una base para mejorar la situación personal, quiénes acceden a ella la valorarán en ese sentido. Es lo que está detrás de la consideración de que los pagos que realiza el sujeto para costear la educación superior, universitaria o técnico profesional, constituyen una inversión, ya que hay una alta correlación entre estos estudios superiores y el ingreso que se obtiene. Aunque nadie lo plantea, es posible generalizar esto a toda la educación, puesto que cada año de estudios que se tenga en cualquier nivel aseguraría mejores ingresos. De la misma manera, se puede sostener que existen intereses particulares de quiénes asumen la función de educadores al margen del Estado. Tales intereses pueden ir desde la propagación de doctrinas de carácter religioso, de determinadas ideologías o perspectivas políticas, hasta la expectativa simple y llana de obtener una ganancia con ello.

Los intereses particulares de quien quiere educarse para obtener un mejor ingreso o mejorar su comprensión del mundo, y de quiénes desean educar para propagar una fe, una ideología u obtener una ganancia, pueden estar presentes en muy diversa medida. Sin embargo, la relación del Estado con la educación, definida como bien público, no se relaciona con el interés particular de los sujetos individuales, sino con el proyecto de sociedad que se quiere desarrollar y los derechos ciudadanos que este Estado reconoce. 
En la medida que el Estado privilegia los intereses particulares del educador y del educado, la educación se transforma efectivamente en un bien de consumo que se rige por las leyes del mercado, por muy imperfecto que sea éste. En la medida que el Estado asume el interés general, la educación es un bien público que se pone a disposición de la comunidad sin distinciones.

Estas aclaraciones son la clave para entender la discusión respecto a privilegiar la libertad de enseñanza o el derecho a la educación. La libertad de enseñanza se entiende desde la perspectiva del mercado, en el cual yo puedo ofrecer diferentes orientaciones, calidades y cantidades del servicio y, a la vez, se puede demandar educaciones también diferentes, estableciéndose un precio de ajuste entre oferta y demanda. Pero este mercado no difiere de otros mercados, en el sentido de que la diferenciación en cuanto al acceso a un bien y a su cantidad y calidad se establece según poder de compra. Por tanto, mientras mayor sea la diferencia de poder de compra mayor será la diferencia en el acceso a bienes y servicios.

Por el contrario, un bien público no está en el mercado y su acceso es en principio igualitario. Las calles, las playas y plazas son bienes públicos pues todos pueden tener acceso a ellos en condiciones de igualdad. Lo mismo se podría señalar para el servicio de registro civil, la iluminación pública, la policía, las campañas de salud pública, etc. No tiene nada de extraño que esos bienes también poco a poco se vayan privatizando, dado que eso es consecuencia del modelo adoptado. En la medida que estos bienes pasan a estar limitados en su acceso por el requerimiento de pago, como ocurre con las vías con peaje, las playas concesionadas o estacionamientos controlados, dejan de ser bienes públicos.

Lo que está ocurriendo con la educación no es algo especial y aislado. Al eliminarse el criterio de un sistema de educación público a través del cual el Estado cumple con la obligación que le impone el derecho a la educación, la educación se regula en el mercado. Al asumir que la educación tiene un precio que debe pagar quien se provee de ella, el bien se privatiza. El hecho de que el Estado esté dispuesto a regalarle plata a los muy pobres y a prestársela a los que no lo sean tanto, para que puedan pagar su educación, no la repone como un derecho, pues éste tendría que ser igualitario. Estas transferencias operan como un acto hacia los desposeídos que puede corresponder a justificaciones de piedad o a cálculo político de descomprimir conflictos, pero es el reconocimiento de que los no favorecidos no tienen derechos, pues necesitan ser apoyados para poder comprar el servicio. La focalización del gasto se basa en la diferencia, no en la igualdad. 
Entre los argumentos que se esgrimen en defensa del sistema de becas y créditos para los más pobres (eufemísticamente denominados menos privilegiados o vulnerables) está el que por este medio se está redistribuyendo el ingreso en favor de estos y se evita una distribución regresiva como sería dar educación gratuita a quien puede pagarla. Casi se afirma que así se estarían produciendo los mismos efectos que la educación gratuita, pues quienes la necesitan tienen los apoyos para efectivamente tenerla.

Esta pretensión, de que el Estado garantiza el derecho a la educación de los que la necesitan y no tienen poder de compra para pagar su precio mediante el sistema de subvenciones, es contradicha en los hechos y en los principios En los hechos, porque la subvención que entrega el Estado para pagar la educación que los sostenedores municipales y particulares entregan se fija en un mínimo que permite financiar una pobre educación, mientras que quiénes tienen más poder de compra pueden adquirir una educación de mayor calidad. En principio, porque el sistema de subvenciones reconoce una desigualdad y no una igualdad frente a la educación. Lo mismo podría plantearse respecto de las becas y créditos, con el agravante, en este último caso, que la deuda contraída prolonga la diferencia económica inicial del supuesto beneficiado. La beca sólo tendría sentido, desde la perspectiva de que la educación es un bien público, si estuviera destinada a mejorar las condiciones de un pobre para poder superar las condiciones económicas que le impiden un mejor desempeño, es decir, becas de mantenimiento.

Naturalmente que para que el Estado cumpla con el derecho a la educación se requieren recursos, pero una cosa es la decisión política de lograr un objetivo que se considera valioso y otra cosa es la implementación para que tal decisión pueda llevarse a cabo. El que recientemente en Chile la propuesta de reforma impositiva de intención redistributiva haya sido exitosamente resistida por el empresariado, demuestra que cualquier alteración del modelo tendrá muy serias dificultades. Al respecto, una frase muy repetida para defender el actual modelo de educación universitaria es que no se puede pretender que con el impuesto que pagan los pobres se financie la educación de los ricos. Más allá del hecho de que sea discutible que sólo los ricos acceden a tal educación (sólo el 5\% de los hogares tiene un ingreso superior a \$1.400.000, que podría con dificultades financiar una arancel universitario), lo escandaloso es el reconocimiento de que los impuestos los pagan los pobres.

Finalmente, habría que aclarar un equívoco recurrente. En la discusión sobre el problema de la educación suele ponerse mucho énfasis en que la educación es el más importante mecanismo de movilidad. Talvez lo sea, pero 
sólo entendido en términos de movilidad individual, puesto que es probable que si alguien adquiere mayor educación que otros puede mejorar su situación económica. Pero la educación por sí sola no es un vehículo de movilidad social. Nadie razonablemente puede esperar que mediante la educación los pobres pasen a ser ricos y los ricos pasen a pobres. Más aún, se puede constatar muy fácilmente que los pobres de ahora tienen muchos más años de educación que los pobres de antes. Los de antes eran analfabetos, los de ahora tienen hasta educación media, en el futuro posiblemente tengan doctorado.

La educación como bien público, como un derecho ciudadano frente al cual el Estado tiene que cumplir con la obligación recíproca, corresponde a la necesidad que tiene toda sociedad de formar laboral y éticamente a sus ciudadanos para que tal sociedad se reproduzca. En tal sentido, como bien público, tiene que cumplir con la exigencia de igualdad de esa esfera y no con la diferenciación propia del mercado. Cuando el Estado no asume la educación como derecho ciudadano, será el mercado el que regule, según el poder de compra, el acceso diferencial a la educación.

La experiencia chilena respecto al deterioro de la educación pública y de la universidad pública tiene el tinte dramático de que se impuso bajo una dictadura y que ha alcanzado niveles inusitados. Sin embargo, no es un dato menor que ese proceso no haya sido revertido durante más de un cuarto de siglo de gobiernos democráticos. Más aún, es posible apreciar que internacionalmente también existe un creciente proceso de privatización de la educación y de las universidades, por más que en algunos casos la defensa de la universidad pública haya sido más exitosa. Si levantamos más el vuelo y apreciamos lo que está ocurriendo en otras esferas, como la previsión, la salud, las vías públicas, los espacios urbanos, el transporte, las comunicaciones, veremos que ahí también se asiste a procesos de privatización. La transferencia de lo público al ámbito de lo privado y a su regulación por el mercado es un proceso propio de la instauración de un nuevo modelo económico y de relaciones sociales. Pero si, como señalamos, la definición de lo público y lo privado sólo la puede realizar el Estado como voluntad de la totalidad social, el problema se planteará en torno a la manera en que se forma esa voluntad del todo. Es aquí precisamente donde pareciera estar derivando la crítica social: al cuestionamiento de cómo se forma esa voluntad, que no pareciera dar cuenta concretamente de los intereses generales. No es raro que últimamente se hable cada vez más de un cuestionamiento a la base de conformación del régimen político democrático en términos de una crisis de representatividad. 
¿Qué pasará con la educación y la igualdad? Es difícil saberlo, pero no cabe duda que su futuro está ligado al futuro de lo público en general y que el futuro de lo público en general lo define la voluntad política.

\section{NOTAS}

1. El presente texto está basado en una conferencia presentada en la Universidad de Costa Rica con motivo de la celebración de su $72^{\circ}$ aniversario el 30 de agosto de 2012. 
